\title{
Ruptura diafragmática traumática diagnosticada preoperatoriamente. A propósito de un caso
}

\section{Traumatic diaphragmatic injury diagnosed preoperatively. A Case report}

\author{
I. González-Pezzat ${ }^{1}$, E. Soto-Pérez de Celis², E. Villanueva-Sáenz ${ }^{1}$
}

\section{RESUMEN}

La ruptura diafragmática representa un reto diagnóstico para los médicos que se enfrentan al manejo de pacientes politraumatizados. Esta lesión, aunque poco común, debe sospecharse en ciertos tipos de accidentes, por lo cual es vital conocer su relación con la cinemática del trauma. En este trabajo presentamos un caso de ruptura diafragmática con herniación intratorácica del contenido abdominal que fue reparada quirúrgicamente. Así mismo hacemos una revisión del cuadro clínico, diagnóstico y tratamiento de esta patología, haciendo hincapié en la importancia de mantener siempre un alto índice de sospecha para lograr un diagnóstico oportuno.

Palabras clave. Diafragma. Hernia diafragmática traumática. Lesiones torácicas. Trauma múltiple. Accidentes tráfico.

\begin{abstract}
Traumatic diaphragmatic rupture represents a diagnostic challenge to any physician dealing with polytraumatized patients. This uncommon injury must be suspected in certain types of accidents, making knowledge of trauma mechanisms vital for its diagnosis. In this paper we present a case of traumatic diaphragmatic rupture with intrathoracic herniation of abdominal contents, which was surgically repaired. We also present a review of the clinical characteristics, diagnosis and treatment of this entity, emphasizing the importance of keeping a high index of suspicion in order to achieve a correct diagnosis.
\end{abstract}

Key words. Diaphragm. Traumatic diaphragmatic hernia. Thoracic lesions. Multiple trauma. Traffic accidents.
1. División de Cirugía. Hospital Ángeles Pedregal. Ciudad de México.

2. Departamento de Medicina Interna. Instituto Nacional de Ciencias Médicas y Nutrición Salvador Zubirán. Ciudad de México.

\section{Correspondencia}

Enrique Soto Pérez de Celis

Camino a Santa Teresa, 890, torre 13, Dpto. 401

La Magdalena Contreras, DF México

E-mail: enriquesotopc@yahoo.com 


\section{INTRODUCCIÓN}

El diafragma es un músculo en forma de domo que divide la región torácica de la abdominal y es el principal motor de la ventilación ${ }^{1}$. La incidencia de ruptura diafragmática es del 0,8-5,8\% de todas las lesiones no penetrantes, ocurriendo en el $2,5-5 \%$ de los traumas abdominales cerrados y en el 1,5\% de los traumas torácicos ${ }^{2}$. La mortalidad en pacientes con ruptura diafragmática es del 5,5 al $51 \%$, siendo la causa más frecuente las lesiones asociadas y las complicaciones intrahospitalarias $^{1}$. En la mayoría de los pacientes con ruptura diafragmática se encuentran lesiones asociadas, principalmente la fractura de la pelvis y el traumatismo craneoencefálico, que varían en frecuencia según el tipo de impacto. El hemidiafragma izquierdo se lesiona en un $50-80 \%$ de los casos, mientras que las lesiones del lado derecho se presentan con menos frecuencia $(12-40 \%)^{3}$. En el caso de un accidente automovilístico, se debe considerar que un choque frontal o lateral que disminuya el tamaño de la cabina de los pasajeros en 30 centímetros o un impacto a una velocidad por lo menos $40 \mathrm{~km} / \mathrm{h}$ asociado a lesiones de órganos abdominales tiene una sensibilidad de entre el 68 y el $89 \%$ para detectar una ruptura diafragmática ${ }^{4}$.

La ruptura diafragmática representa un reto diagnóstico para cualquier médico que se enfrenta a pacientes politraumatizados. Para diagnosticarla de forma oportuna se requiere mantener un alto índice de sospecha y realizar los estudios de imagen necesarios para comprobarla. Es de vital importancia conocer la cinemática del trauma ya que, en la mayoría de los casos, la etiología de la ruptura es un impacto vehicular de alta energía con fuerzas frontales y latera- les. Es muy común encontrar lesiones asociadas, de las cuales las más frecuentes son las lesiones torácicas, en hígado, bazo, las fracturas de pelvis y el traumatismo craneoencefálico.

\section{CASO CLÍNICO}

Presentamos el caso de una mujer de 53 años que ingresó a nuestra sala de urgencias después de haberse visto involucrada en un accidente automovilístico a una velocidad aproximada de $50 \mathrm{~km} / \mathrm{h}$ con impacto lateral izquierdo. A su llegada presentaba dolor intenso en la región cervical y dorsolumbar, disnea y dolor en el hemitórax izquierdo. Así mismo refería dolor abdominal localizado en la fosa iliaca derecha, además de dolor a la movilización de la cadera. A la exploración se observó disminución de la movilidad del hemitórax izquierdo. La auscultación del tórax reveló ausencia del murmullo vesicular y de las vibraciones vocales en la región infraescapular izquierda. El abdomen se encontraba distendido, sin heridas penetrantes, muy doloroso a la palpación, con datos de irritación peritoneal y con ausencia de ruidos peristálticos. Se realizó una ecografía abdominal que no reportó líquido libre. Se indicó una radiografía de tórax y se observaron asas intestinales en el hemitórax izquierdo (Fig. 1A). Se decidió realizar una tomografía donde se observó una ruptura diafragmática izquierda con el estómago, el colon y el bazo en situación intratorácica (Fig. 1B). Se documentó una contusión pulmonar izquierda y fractura de pelvis bilateral. La paciente se sometió a una laparotomía exploradora, reduciéndose el contenido intratorácico y observándose una ruptura diafragmática de aproximadamente $10 \mathrm{~cm}$ (Fig. 2). No se encontraron lesiones asociadas, por lo que se reparó el defecto con sutura continua de prolene 1-0 y se reforzó con una malla de prolene. Se colocó un tubo pleural en el pulmón afectado. La evolución de la paciente fue favorable y no presentó complicaciones postquirúrgicas. 
Figura 1. A. Radiografía de tórax mostrando órganos abdominales herniados en el interior del hemitórax izquierdo (cabeza de flecha) y desviación traqueal. B. Tomografía computada de tórax con herniación de órganos abdominales en la cavidad torácica (flecha).

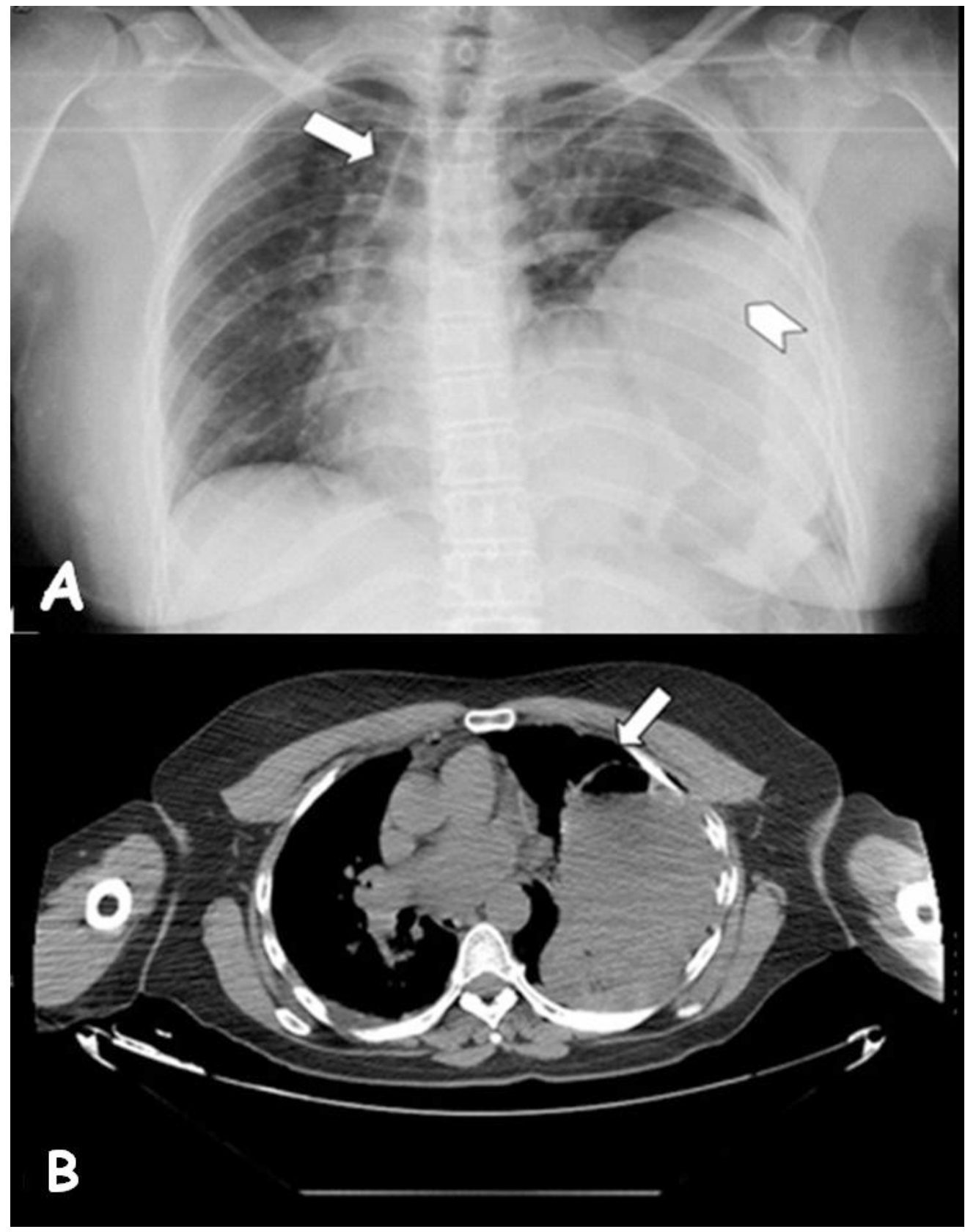


Figura 2. Aspecto de la ruptura diafragmática después de la reducción del contenido abdominal.

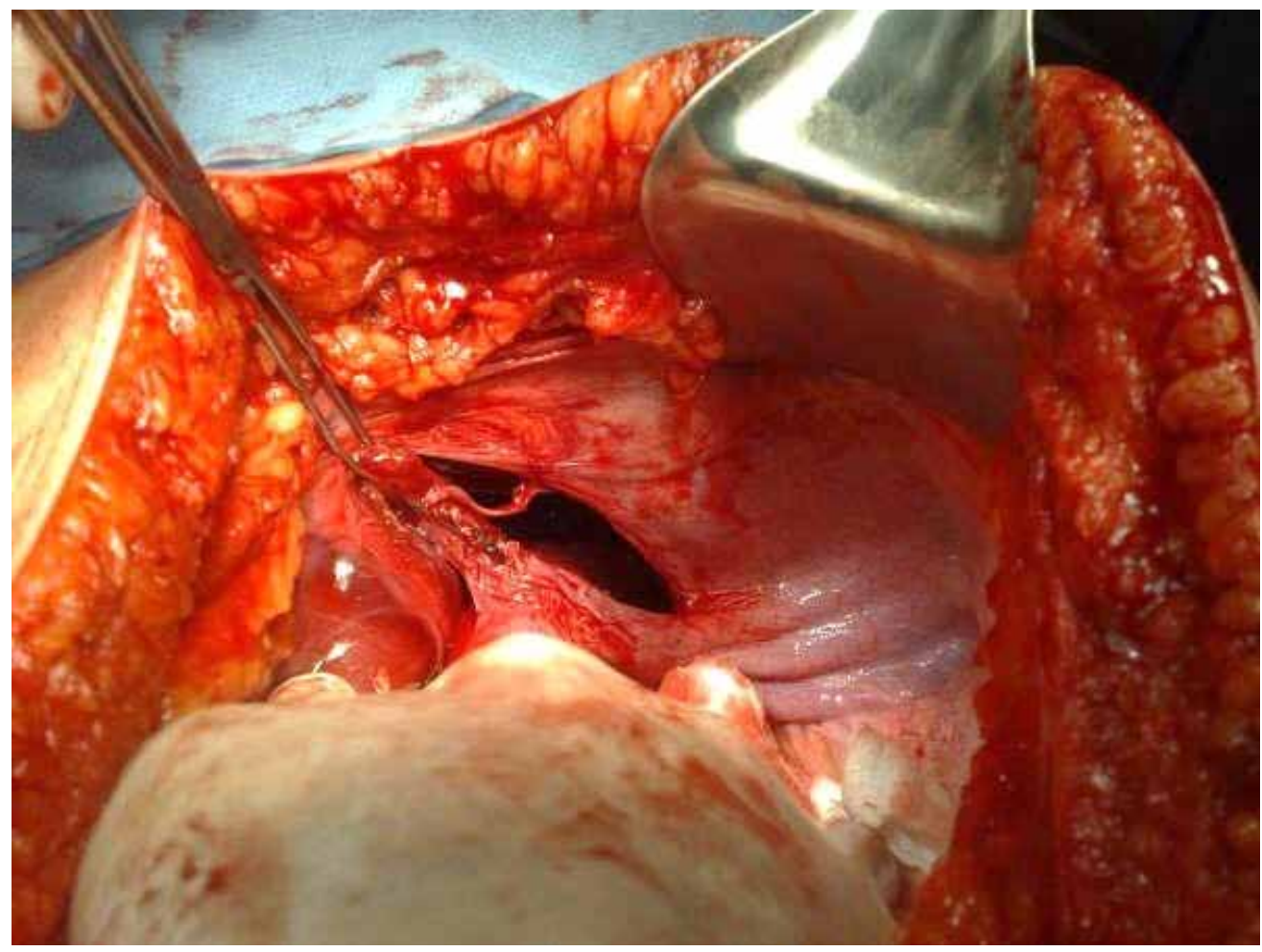

\section{DISCUSIÓN}

El diagnóstico clínico de la ruptura diafragmática es extremadamente difícil. Algunos datos clínicos comunes son la disnea, cianosis, dolor en los hombros y dolor torácico. La presentación clínica más frecuente es la dificultad respiratoria, secundaria al colapso pulmonar por los órganos herniados. Los órganos que se hernian con más frecuencia son el epiplón, seguido del estómago, el bazo, el colon y con menor frecuencia el hígado ${ }^{5}$. La auscultación de ruidos intestinales en el tórax y la disminución o ausencia de ruidos respiratorios pueden ser sugestivas ${ }^{6}$.

En lo que a estudios de imagen se refiere, la radiografía de tórax logra diagnosticar de un $8,3-25 \%$ de rupturas diafragmáticas en las primeras 24 horas $^{5}$. La tomografía computarizada permite la identificación de lesiones asociadas y de órganos herniados, teniendo la ventaja de delinear con gran precisión la presencia de lesiones vasculares $^{3}$. Otros métodos diagnósticos útiles son el ultrasonido y la resonancia magnética. A pesar de contar con diversos métodos radiológicos, es importante mencionar hasta el $45 \%$ de los pacientes no son diagnosticados hasta que llegan a la sala de operaciones $^{6}$.

Una vez establecido el diagnóstico, la reducción quirúrgica es el único método terapéutico para lograr una reparación definitiva. Las rupturas diafragmáticas del lado izquierdo se abordan mejor por la vía abdominal, aunque algunos autores prefieren el abordaje torácico, realizando una laparotomía sólo cuando es necesario. Por otra parte, las lesiones derechas pueden ser manejadas de mejor forma mediante una toracotomía ${ }^{3}$. La reparación del defecto se puede realizar de directamente mediante una sutura continua o puntos 
separados con material no absorbible; o alternativamente se puede colocar una malla de prolene, sobretodo cuando el defecto es muy grande ${ }^{7}$. La experiencia en el abordaje laparoscópico de este tipo de lesiones aún es poca, aunque se han reportado buenos resultados con su uso. Entre las ventajas teóricas de dicho abordaje se encuentran la mínima invasión, la posibilidad de extubar precozmente a los pacientes y la posibilidad de realizar una exploración laparoscópica para descartar lesiones asociadas ${ }^{8}$. En cuanto al momento de la indicación quirúrgica, es importante mencionar que la reparación de la ruptura diafragmática puede diferirse siempre y cuando no existan otras indicaciones de cirugía urgente, lo cual permitiría estabilizar primero las lesiones craneales o pulmonares concurrentes ${ }^{9}$.

La ruptura diafragmática postraumática es una lesión poco frecuente, por lo que es necesario un alto índice de sospecha para poder diagnosticarla antes de someter al paciente a una intervención. Se debe tomar en cuenta que la mortalidad de estos pacientes se encuentra íntimamente relacionada con las complicaciones postquirúrgicas, por lo que es necesario un adecuado abordaje inicial para planear de manera adecuada el manejo transoperatorio.

\section{BIBLIOGRAFÍA}

1. Clint W. SLIKER MD. Imaging of diaphragm injuries. Radiol Clin North Am 2006; 44: 199-211.

2. AraK T, Solheim K, Pillgram-Larsen J. Diaphragmatic injuries. Injury 1997; 28: 113-117.

3. Hacilbrahimoglu K, Solak O, Olcmen A, Bedirhan M, Solmazer N, Gurses A. Management of traumatic diaphragmatic rupture. Surg Today 2004; 34: 111-114.

4. Reiff DA, McGwin G, Metzger J. Identifying injuries and motor vehicle collision characteristics that together are suggestive of diaphragmatic rupture. J Trauma 2002; 53: 1139-1145.

5. ERen M, Kantarci M, OKur A. Imaging of diaphragmatic rupture after trauma. Clin Radiol 2006; 61: 467-477.

6. Chughtai T, McSyed A, Sharkey P, Lins M, Rizoli S. Update on managing diaphragmatic rupture in blunt trauma: a review of 208 consecutive cases. Can J Surg 2009; 52: 110-119.

7. Reda E, Al-Refaie E, Awad E, Mokbel E. Blunt traumatic diaphragmatic rupture: a retrospective observational study of 46 patients Interact CardioVasc Thorac Surg 2009; 9: 45-49.

8. García-NaVArRo A, Villar-Del Moral JM, MuffaKGranero K, Palomeque-Jiménez A, Mansilla-RoseLLÓ A, GARROTE-LARA D et al. Rotura traumática del diafragma. Cir Esp 2005; 77: 105-107.

9. Cerón Navarro Jeñalver Cuesta JC, Padilla Alarcón J, Jordá Aragón Q, Escrivá Peiró J, Calvo Medina V et al. Rotura diafragmática traumática. Arch Bronconeumol 2008; 44: 197-203. 
